\title{
Strategies for antenatal detection of Down's syndrome
}

\author{
Jonathan P Wyllie, R John Madar, Michael Wright, John Burn, Christopher Wren
}

\begin{abstract}
Aim-To predict the effect of maternal serum screening and fetal echocardiography on the birth prevalence of Down's syndrome.

Methods-The outcome of all Down's syndrome pregnancies in the Northern Health Region between 1985 and 1991 was retrospectively ascertained. The number and outcome of all Down's syndrome pregnancies were used to define a theoretical population which would exist in the absence of screening. Published reports were used to predict the effects of screening strategies.

Results-Down's syndrome was identified in 412 pregnancies of which $315(76 \%)$ resulted in live birth. A theoretical population with no antenatal screening would be expected to produce 31 stillbirths and $381(92 \%)$ live births affected by Down's syndrome. In the same population a programme of maternal serum screening and fetal echocardiography would lead to 155 and 14 terminations, respectively, and when combined, would reduce affected live births to $229(56 \%)$.

Conclusions-Even if maternal serum screening and fetal echocardiography achieve their predicted potential, around half of all pregnancies affected by Down's syndrome will result in live born babies. (Arch Dis Child 1997;76:F26-F30)
\end{abstract}

Keywords: Down's syndrome; screening; maternal serum screening.

Down's syndrome is the commonest chromosomal abnormality in live born babies. It affects about 1 in 700 pregnancies at 20 weeks of gestation. Antenatal screening and termination of affected pregnancies have been available for several years. Until recently screening relied on the increase in incidence of Down's syndrome with maternal age, so that amniocentesis was offered only to mothers over $35^{1}$ or $36^{23}$ or $37^{45}$ years of age. However, only $20-30 \%$ of mothers are aged 35 years or older, ${ }^{6}$ so a screening programme based on age related amniocentesis has a limited impact.

More recently maternal serum concentrations of $\alpha$-fetoprotein, human chorionic gonadotrophin, and oestriol have been used in addition to maternal age to identify pregnancies at "increased risk." To restrict amniocentesis to about $5 \%$ of all pregnancies, different units have chosen thresholds of increased risk varying from 1 in 190 to 1 in $270 .^{16}$
Mothers judged to be at "increased risk" are offered amniocentesis to confirm or exclude the presence of Down's syndrome, but those thought to be "not at increased risk" are not. Such a screening programme is often misunderstood despite extensive attempts at counselling and explanation. ${ }^{78}$ Serum screening works better in older mothers, although they are no more likely to enrol for it than younger mothers and they may be less likely to proceed to amniocentesis faced with a result predicting an increased risk. ${ }^{19}$

Down's syndrome may also be suspected from abnormalities shown by ultrasound examination. Identification of a complete atrio-ventricular septal defect on fetal echocardiography strongly suggests the diagnosis, but the prospective diagnosis rate in population studies is not high. ${ }^{10}$ Other abnormalities (duodenal atresia, nuchal skin thickening, femoral shortening, etc) are associated with Down's syndrome but are not useful in screening at present. ${ }^{11}$

This study set out to investigate the likely cumulative effect of screening for Down's syndrome using maternal serum screening and fetal echocardiography. All cases of Down's syndrome beyond the 20 th week of pregnancy were identified in a defined population. Affected pregnancies which were terminated as a result of the various screening programmes in use in the observed population were "reallocated" to have been live born or stillborn in the same proportions as in the observed population, to produce a theoretical population which would exist in the absence of any screening. This theoretical population was used to predict the likely effect of screening.

\section{Methods}

OBSERVED POPULATION

The former Northern Health Region had a population of 3 million 100000 and close to 40000 live births every year. With the exception of the health district of South Cumbria, there was no cross border referral of infants with Down's syndrome or congenital heart disease. This study was therefore based on the remaining 15 health districts within the region. The region contains the Northern Regional Survey of Perinatal, Neonatal, and Infant Mortality and the Northern Regional Fetal Abnormality Survey, both of which are population based and whose methodologies have been validated and published. ${ }^{12}{ }^{13}$ All cases of Down's syndrome in and beyond the 20 th week of pregnancy in 1985-91 were identified from the two regional surveys; from the 
Table 1 Assumptions made from published data in predicting the effects of screening strategies

\begin{tabular}{lccc}
\hline & $\begin{array}{l}\text { Age related } \\
\text { amniocentesis }\end{array}$ & $\begin{array}{l}\text { Maternal serum } \\
\text { screening }\end{array}$ & $\begin{array}{l}\text { Fetal } \\
\text { echocardiography }\end{array}$ \\
\hline Assumed uptake of test & $50^{\star}$ & 81 & 91 \\
Assumed detection rate & 100 & $61 / 91^{\dagger}$ & 15 \\
Assumed amniocentesis rate & 100 & $83 / 59^{\dagger}$ & 75 \\
Assumed termination rate & 50 & 90 & 100 \\
Effective reduction & 33 & $37 / 39^{\dagger}$ & 10 \\
Proportion of population screened $\ddagger$ & 17 & 100 & 34 \\
Total effective reduction $\dagger \dagger$ & & $37 / 39^{\dagger}$ & 3 \\
\hline
\end{tabular}

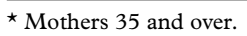

t Under 35/35 and over.

$\ddagger$ ie \% of mothers 35 and over or \% of fetuses with CHD.

t† Allowing for prevalence of maternal age $\geq 35$ or prevalence of congenital heart disease in the fetus. of Perinatal, Neonatal, and Infant Mortality. These were added to the number of live births to give the total number of pregnancies at 20 weeks of gestation.

The prevalence and spectrum of congenital heart disease in live born babies with Down's syndrome were identified from the regional paediatric cardiology database. Unfortunately, as previous reports have suggested, ${ }^{14}$ too few terminated and stillborn fetuses underwent a sufficiently detailed necropsy for data about the prevalence of congenital heart disease in stillbirths to be useful. Congenital heart disease in live born babies was classified by its potential detectability on antenatal four chamber screening at 18 weeks of gestation. ${ }^{14}$

regional cytogenetics laboratories at Newcastle upon Tyne, Middlesbrough, and Seascale; and from the regional paediatric cardiology department at Freeman Hospital. These years were chosen because they predated more widespread introduction of fetal echocardiography and maternal serum screening. Cytogenetic confirmation of all cases was available. All cases were validated using maternal, infant, and pregnancy details and cross checked with the Office of Population Censuses and Surveys (National Statistics Office) to avoid duplication and exclude misclassification. The maternal age at diagnosis, method of diagnosis, and outcome were noted.

Information on antenatal diagnosis of Down's syndrome and subsequent termination of pregnancy was obtained from the regional cytogenetics laboratories and from the Fetal Abnormality Survey. The total number of pregnancies and total number of live births for the population were identified from the Office of Population Censuses and Surveys. All terminated pregnancies, stillbirths (28 weeks to term) and late miscarriages (20-27 weeks) were identified from the Northern Regional Survey

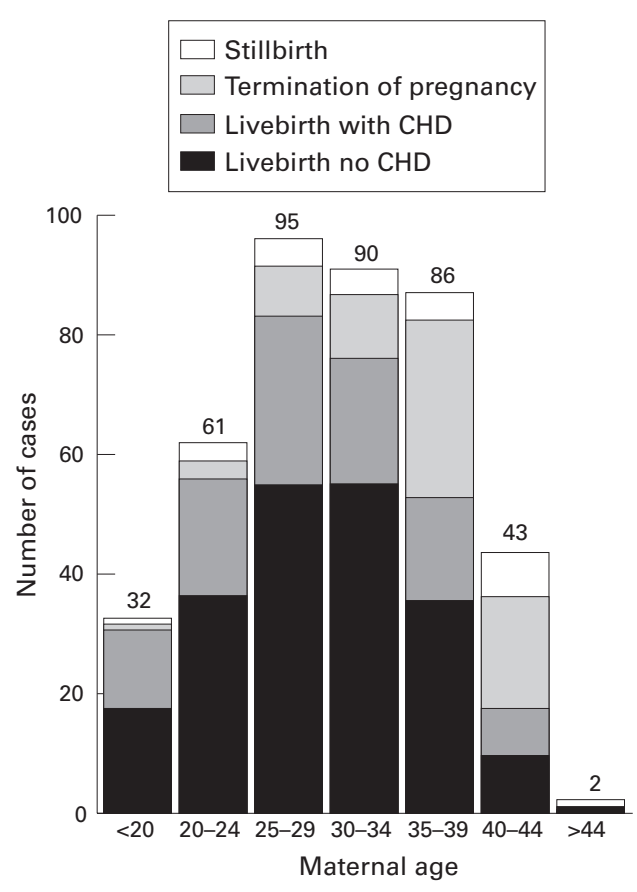

Figure 1 Total cases and outcomes of pregnancies related to maternal age. CHD: congenital heart disease.

\section{THEORETICAL POPULATION AND PROJECTED EFFICACY OF SCREENING}

The observed population includes live born babies with Down's syndrome, stillborn babies with Down's syndrome, and pregnancies that were terminated as a result of age related amniocentesis, maternal serum screening leading to amniocentesis, or fetal echocardiography leading to amniocentesis. To derive the theoretical underlying population in the absence of screening, those pregnancies that were terminated were reallocated to be live born or stillborn in the same proportions as in the observed population. The theoretical population was used to predict the effectiveness of the following screening tests.

\section{Amniocentesis}

The effect of age related amniocentesis was predicted by assuming it would be offered only to mothers of 35 years and older (table 1) and that the uptake of the amniocentesis would be $50 \%,{ }^{9}{ }^{15}$ although this is higher than in some other reports. ${ }^{4}$

\section{Maternal serum screening}

To predict the effect of maternal serum screening it is important to realise that the overall effectiveness will be the product of the proportions of mothers: enrolling for the test; being detected by the test; proceeding to amniocentesis to confirm the diagnosis; undergoing termination of pregnancy. The present performance of maternal serum screening was obtained by using the highest published figures and assuming that $81 \%$ of mothers would undergo testing, ${ }^{16}$ a $61 \%$ detection in mothers under 35 , and $91 \%$ in mothers 35 or over (70\% overall), ${ }^{16}$ that $83 \%$ of mothers under 35 and $59 \%$ of those 35 or over $(79 \%$ overall) would proceed to amniocentesis, ${ }^{1}$ and that $90 \%$ of confirmed affected pregnancies would be terminated. ${ }^{9}$ The cumulative effect of these figures means that the overall detection rates are $37 \%$ and $39 \%$ for mothers under 35, and 35 and over, respectively.

\section{Fetal echocardiography}

Most babies with Down's syndrome do not have congenital heart disease, but more than half of those who do have some form of atrioventricular septal defect that is potentially detectable on a routine antenatal four chamber 
Table 2 Derivation of the theoretical population from the observed population

\begin{tabular}{llll}
\hline & $\begin{array}{l}\text { Maternal } \\
\text { aget }\end{array}$ & $\begin{array}{l}\text { Observed } \\
\text { population }\end{array}$ & $\begin{array}{l}\text { Theoretical population } \\
\text { (no fetal diagnosis) }\end{array}$ \\
\hline Total cases & $<35$ & 280 & 280 \\
& $\geq 35$ & 132 & 132 \\
Terminations & Total & 412 & 412 \\
& $<35$ & 24 & 0 \\
& $\geq 35$ & 50 & 0 \\
Stillbirths & Total & 74 & 0 \\
& $<35$ & 12 & 13 \\
Live births & $\geq 35$ & 11 & 18 \\
& Total & 23 & 31 \\
Liveborn cardiac abnormality total & $<35$ & 244 & 266 \\
Liveborn cardiac abnormality detectable & $\geq 35$ & 71 & 115 \\
* & \multicolumn{4}{c}{ Total } & 315 (76\%) & 383 (93\%) \\
& \multicolumn{4}{c}{55} & 67 \\
\hline
\end{tabular}

(see text). The missing three ages were assumed to be $<35$ in 2 and $\geq 35$ in 1 .

scan. ${ }^{417}$ Identification of such an abnormality may be an indication for amniocentesis. To predict the effect of a screening programme using cardiac ultrasonography, it was assumed that $91 \%$ of pregnancies are screened at an appropriate gestation, ${ }^{18}$ that in $15 \%$ of cases of Down's syndrome with a cardiac malformation the defect would be recognised, ${ }^{10}$ and that in $75 \%$ this would lead to amniocentesis and termination of pregnancy. ${ }^{17}$

\section{Combined maternal serum screening and fetal echocardiography}

The effects of maternal serum screening and fetal echocardiography are not simply additive as there is an overlap of two or more screening strategies. We have assumed that maternal serum screening is unaffected by the presence or absence of a cardiac malformation and that the prevalence of congenital heart disease is not affected by maternal age. The likely prevalence of congenital heart disease was computed for cases undetected by maternal serum screening and the effect of echocardiographic diagnosis on cardiac malformation predicted as outlined (table 1).

Statistical analysis was limited to comparison of the prevalence of stillbirths in mothers younger than 35 years and in those 35 years and older using a $\chi^{2}$ test.

\section{Results}

OBSERVED POPULATION

There were 285681 pregnancies of at least 20 weeks of gestation between 1985-91, of which 283521 resulted in live birth. In the same period there were 412 pregnancies with Down's syndrome present at 20 weeks of gestation (1.44 per 1000 pregnancies) of which 74 (18\%) were terminated, 23 ( $7 \%$ of nonterminated pregnancies) ended with stillbirth, and $315(76 \%)$ resulted in live birth $(1.11$ per 1000 live births). The pregnancies which were terminated were diagnosed by age related amniocentesis in 40 pregnancies, as a result of fetal echocardiography or ultrasonography in 10 pregnancies, and after maternal serum screening or amniocentesis for other reasons in 24 cases.

The maternal age was known in 409 pregnancies (99\%), and the maternal age distribution at diagnosis is summarised in fig 1 . Mothers were younger than 35 years in 278 (68\%) pregnancies and in $242(77 \%)$ live births. Stillbirths were more common in older mothers- $14 \%$ in mothers aged 35 or over compared with $5 \%$ in mothers younger than 35 years $(0.05<\mathrm{P}<0.1)$. The three cases in which maternal age was unknown (all live born) were assumed to be under 35 in two and 35 or over in one (table 2). There was no significant difference in the birth prevalence of congenital heart disease and Down's syndrome with increasing maternal age.

Congenital heart disease was present in 106 of 315 live born babies (34\%). The malformation was a complete atrio-ventricular septal defect in 45 cases, a partial atrio-ventricular septal defect in 10, a ventricular septal defect in 26 , a patent ductus arteriosus in 10 , an atrial septal defect in seven, tetralogy of Fallot in four, coarctation of the aorta in two and complex in two cases. Of these, only atrioventricular septal defects are easily detectable by routine four chamber antenatal ultrasound screening. ${ }^{14} \mathrm{~A}$ further 13 cases of cardiac malformation were found in terminated fetuses and four in stillborn babies. However, too few terminated and stillborn fetuses underwent a sufficiently detailed necropsy to include these cases in the analysis.

\section{THEORETICAL POPULATION}

To derive a theoretical population which would exist if no screening was available, the 74 pregnancies terminated in the observed population were allocated to live birth (66) or stillbirth (8) in the same proportions seen in each maternal age group (table 2). The criteria set out in table 1 were applied to this theoretical population to predict the effects of various screening strategies.

Age related amniocentesis

Age related amniocentesis with a 50\% uptake and termination of all affected pregnancies in mothers aged 35 or over would result in $16 \%$ of pregnancies being terminated and $78 \%$ producing live born babies (table 3).

\section{Maternal serum screening and fetal echocardiography}

Maternal serum screening alone would result in $38 \%$ of pregnancies being terminated and $58 \%$ live births (table 3). Fetal echocardiogra-

Table 3 Predicted effect of screening strategies on outcome of pregnancy

\begin{tabular}{lllllll}
\hline & $\begin{array}{l}\text { Observed } \\
\text { population }\end{array}$ & $\begin{array}{l}\text { Theoretical } \\
\text { population }\end{array}$ & Amniocentesis & $\begin{array}{l}\text { Maternal serum } \\
\text { screening }\end{array}$ & $\begin{array}{l}\text { Fetal } \\
\text { echocardiography }\end{array}$ & Combined \\
\hline Total cases & 412 & 412 & 412 & 412 & 412 & 412 \\
Terminations & 74 & 0 & 67 & 155 & 14 & 164 \\
Stillbirths & 23 & 31 & 22 & 19 & 30 & 19 \\
Live births & $315(76 \%)$ & $389(92 \%)$ & $323(78 \%)$ & $238(58 \%)$ & $368(89 \%)$ & $229(56 \%)$ \\
\hline
\end{tabular}


Table 4 Projected numbers of live born babies with Down's syndrome for various screening strategies

\begin{tabular}{lll}
\hline & Per 100000 pregnancies & UK per year \\
\hline Total Down's pregnancies & 144 & 1080 \\
Live births/observed population & 111 & 833 \\
Live births/amniocentesis $>$ 35 years & 115 & 863 \\
Live births/maternal serum screening & 85 & 638 \\
Live births/maternal serum screening + fetal & 81 & 608 \\
$\quad$ echocardiography & &
\end{tabular}

phy alone would result in 3\% of pregnancies being terminated while $89 \%$ would result in live births (table 3 ). The combined effect of maternal serum screening and fetal echocardiography would produce a termination rate of $40 \%$, while $56 \%$ of affected pregnancies would result in live born babies (table 3 ).

Table 4 translates these predictions into rates per 100000 pregnancies and also extrapolates them to the equivalent of the UK population for one year.

\section{Discussion}

This study shows that even if optimistic forecasts of the combined effect of maternal serum screening and fetal echocardiography prove to be accurate, over half of all pregnancies affected by Down's syndrome will result in live born babies. This prediction has major implications for the provision of resources for people with Down's syndrome, whose life expectancy is increasing..$^{19}$ Despite widespread introduction of screening in pregnancy, it is wrong to assume that the population of people with Down's syndrome will be significantly reduced.

Screening for Down's syndrome during pregnancy could originally be achieved only by amniocentesis. The increasing risk of Down's syndrome with increasing maternal age meant that screening was offered only to mothers of 35,36 , or $37^{5}$ years of age and older. However, only a third to half of mothers offered screening elect to undergo amniocentesis ${ }^{415}$ so such screening is relatively ineffective in population terms. Using the maternal age distribution in our population, we estimated that a $50 \%$ uptake of amniocentesis by mothers 35 years or older would detect only $16 \%$ of all Down's pregnancies. Recent studies ${ }^{1520}$ have highlighted the upward trend in maternal age and this phenomenon will affect age related amniocentesis and all other screening programmes where maternal age is a factor. It has recently been suggested ${ }^{21}$ that maternal serum screening should replace age related amniocentesis. Such a proposal would reduce the detection rate in older mothers who would otherwise have undergone amniocentesis but would probably increase the overall detection rate (and reduce the amniocentesis rate) in this age group. $^{21}$

The fact that maternal serum screening is a test for the risk of Down's syndrome rather than a diagnostic test means it is widely misunderstood..$^{78}$ Because serum screening is based on maternal age, there is a differential performance, with a higher detection rate in older mothers. Older mothers may be less likely to volunteer for screening, however, and are less likely to proceed to amniocentesis if the screening test result predicts an increased risk. ${ }^{19}$

We have used only published figures to predict the effect of various screening programmes on a population. Predictions were mainly based on reports by Haddow et $a l^{1}$, Wald et $a l^{9}{ }^{9}$ and Crossley et al. ${ }^{16}$ Haddow et al compared detected cases with predicted cases but did not ascertain real false negative results so the efficacy of screening could only be estimated. ${ }^{1}$ They did not investigate the effect of maternal age on the efficacy of screening. Wald et al used 37 years rather than 35 years as their definition of older mothers. ${ }^{9}$ Their favouring of predicted over observed results has caused controversy. Neither group gives details of the denominator population or of the relative uptake of screening in younger and older mothers. Crossley et $a l$, in a later report, found a higher uptake and a higher detection rate. ${ }^{16}$ They gave no details of uptake of testing or of the decision to proceed to amniocentesis in relation to maternal age, nor did they detail the termination rate once the diagnosis had been confirmed.

When considering the effect of maternal serum screening, it is important to take into account the multiplying effects of the proportions undergoing testing, the detection rate, the amniocentesis rate, and the termination rate. Although detection rates are widely quoted, their effect is reduced by incomplete uptake of testing and by the fact that not all women at increased risk proceed to amniocentesis and then to termination of pregnancy. Thus in the figures we have used an overall detection rate of $70 \%$ would translate to an effective reduction in live born Down's syndrome of only $40 \%$ (because of $81 \%$ uptake of the test, $79 \%$ proceeding to amniocentesis, and $90 \%$ of confirmed cases going on to termination of pregnancy). In the report by Crossley et al -with a detection rate of $70 \%$ - only 21 of 50 cases of Down's syndrome in the whole population studied ( $42 \%)$ were diagnosed as a result of maternal serum screening. ${ }^{16}$

Fetal echocardiography can lead to suspicion of Down's syndrome by detecting complete atrio-ventricular septal defect. However, only $34 \%$ of cases have congenital heart disease, of which $52 \%$ are potentially detectable on routine four chamber screening. ${ }^{14}$ In this study we have assumed that $15 \%$ of detectable cases would be recognised-as reported in the only population based study of the detection of heart disease in fetuses with Down's syndrome of which we are aware, in which the detection rate was $12.5 \%$ for atrio-ventricular septal defects and $15 \%$ overall. $^{10}$ Even a $50 \%$ detection rate would translate to a reduction in affected live births of only $12 \%$ because of the effect of the proportion of Down's syndrome babies with congenital heart disease, the proportion of detectable cardiac malformations, the amniocentesis and termination rates, and overlap with maternal serum screening. Other abnormalities detected by ultrasound screening, such as excess nuchal fold thickness or reduced femur length, may lead to diagnosis 
of Down's syndrome in individual cases but have not yet been shown to be effective in screening. ${ }^{11}$

To predict the cumulative effect of screening with fetal echocardiography and maternal serum screening, we have taken into account the overlap of the different screening strategies as the same case cannot be detected twice. Thus the combined effect of the two screening strategies is less than the sum of the individual methods. The effect of any additional screening strategy would be reduced by the performance of screening programmes already in use.

Another limit on the potential effect of screening programmes is the willingness of purchasing authorities to pay for them. A recent study by Oxfordshire Health Authority concluded that the most cost effective programme would be obtained by offering serum screening only to mothers older than 29 years. ${ }^{22}$

To produce a theoretical population which would exist in the absence of screening we took into account the fact that $7 \%$ of affected pregnancies beyond 20 weeks of gestation would result in stillbirth rather than live birth. This may be an underestimate. Although the necropsy rate in stillbirths is high, it is not always possible to recognise the phenotype of Down's syndrome so some cases may be missed. Hook et al reported an extremely high fetal death rate after diagnosis of Down's syndrome, ${ }^{24}$ but because of significant methodological problems in their report and the very small numbers, we believe our estimate is closer to reality.

Down's syndrome is the commonest congenital cause of severe mental retardation. ${ }^{24}$ There will continue to be considerable interest in, and pressure for, screening in early fetal life. The widespread introduction of maternal serum screening will produce a measurable if not dramatic increase in the number of cases detected. At present it seems likely that more than half the cases will go undetected and will be live born. Further developments may include the possibility of karyotyping fetal cells recovered from maternal blood sampling, but the introduction of such a screening programme is not imminent. Our predictions of the likely numbers of Down's syndrome babies born in the population in the near future have major implications for parents, paediatricians, paediatric cardiologists, and those responsible for health care provision for Down's syndrome patients in the population.
1 Haddow JE, Polamaki GE, Knight GJ, Williams J, Pulkkinen A, Canick JA, et al. Prenatal screening for Down's syndrome with use of maternal serum markers. $N$ Engl f Med 1992;327:588-93.

2 Beekhuis JR, Wolf BTHM, Mantingh A, Heringa MP. The influence of serum screening on the amniocentesis rate in women of advanced maternal age. Prenat Diagn 1994;14:199-202

3 Platt LD, Carlson DE. Prenatal diagnosis - when and how? N Engl F Med 1992;327:367-8.

4 Youings S, Gregson N, Jacobs P. The efficacy of maternal age screening for Down's syndrome in Wessex. Prenat Diagn 1991;11:419-25.

5 Wald N, Wald K, Smith D. The extent of Down's syndrome screening in Britain in 1991. Lancet 1992;340:494.

6 Pauker SP, Pauker SG. Prenatal diagnosis - why is 35 a magic number? N Engl F Med 1994;330:1151-2.

7 Smith DK, Shaw RW, Marteau T. Informed consent to undergo serum screening for Down's syndrome: The gap undergo serum screening for Down's syndrome:
between policy and practice. BMF 1994;309:776.

8 Green JM. Serum screening for Down's syndrome: Experiences of obstetricians in England and Wales. BMF 1992;309:769-72

9 Wald NJ, Kennard A, Densem JW, Cuckle HS, Chard T, Butler L. Antenatal maternal serum screening for Down's syndrome: results of a demonstration project. $B M \mathcal{F}$ 1992;305:391-4.

10 Stoll C, Dott B, Alembik Y, Roth M. Evaluation of routine prenatal ultrasound examination in detecting fetal chromosomal abnormalities in a low risk population. Hum Genet 1993;91:37-41.

11 Lockwood CJ, Lynch L, Berkowitz RL. Ultrasonographic screening for the Down syndrome fetus. Am $\mathcal{f}$ Obstet Gynecol 1991;165:349-52.

12 Northern Regional Health Authority Co-ordinating Group. Perinatal mortality: a continuing collaborative regional survey. BMF 1984;288:1717-20.

13 Northern Regional Survey Steering Group. Fetal abnormality: an audit of its recognition and management. Arch Dis Child 1992;67:F770-4

14 Abu-Harb M, Wyllie J, Hey E, Richmond S, Wren C. Antenatal diagnosis of congenital heart disease and Down's syndrome: the potential effect on the practice of paediatric cardiology. Br Heart f 1995; 74:192-8.

15 Cornel MC, Breed ASPM, Beekhuis JR, Meerman GJ, Kate LP. Down syndrome: effects on demographic factors and prenatal diagnosis on the future livebirth prevalence. Hum Genet 1993;92:163-8.

16 Crossley JA, Aitken DA, Berry E, Connor JM. Impact of a regional screening programme using maternal serum $\alpha$ fetoprotein (AFP) and human chorionic gondotrophin (hCG) on the birth incidence of Down's syndrome in the west of Scotland. F Med Screen 1994;1:180-3.

17 Sharland GK, Lockhart SM, Chita SK, Allan LD. Factors influencing the outcome of congenital heart disease influencing the outcome of congenital heart
detected prenatally. Arch Dis Child 1991;66:284-7.

18 Wyllie JP, Wren C, Hunter S. Screening for fetal cardiac malformations. Br Heart f 1994;71(suppl):20-7.

19 McGrother CW, Marshall B. Recent trends in incidence, morbidity and survival in Down's syndrome. F Mental Deficiency Res 1990;34:49-57.

20 Alberman E, Mutton D, Ide R, Nicholson A, Bobrow M. Down's syndrome births and pregnancy terminations in 1989 to 1993: preliminary findings. Br F Obstet Gynaecol 1995;102:445-7.

21 Haddow JE, Palomaki GE, Knight GJ, Cunningham GC, Lustig LS, Boyd PA. Reducing the need for amniocentesis in women 35 years of age and older with serum markers for screening. N Engl F Med 1994;330:1114-8.

22 Fletcher J, Hicks NR, Kay JDS, Boyd PA. Using decision analysis to compare policies for antenatal screening for Down's syndrome. BMf 1995;311:351-6.

23 Hook EB, Topol BB, Cross PK. The natural history of cytogenetically abnormal fetuses detected at midtrimester amniocentesis which are not terminated electively: new data and estimates of excess and relative risk of late fetal death associated with $47,+21$ and some other abnormal death associated with $47,+21$ and some other

24 Matilainen R, Airaksinen E, Mononen T, Launiala K, Kaariainen R. A population-based study on the causes of mild and severe mental retardation. Acta Paediatr 1995;84:261-6. 